\title{
Detecção de áreas agrícolas em tempo quase real com imagens Modis
}

\author{
Isaque Daniel Rocha Eberhardt(1), Alfredo José Barreto Luiz ${ }^{(2)}$, Antonio Roberto Formaggio(1) \\ e leda Del'Arco Sanches ${ }^{(1)}$
}

\begin{abstract}
(1)Instituto Nacional de Pesquisas Espaciais, Avenida dos Astronautas, no 1.758, Jardim da Granja, Caixa Postal 515, CEP 12227-010 São José dos Campos, SP, Brasil. E-mail: isaquedanielre@hotmail.com, formag@dsr.inpe.br, ieda@dsr.inpe.br (2)Embrapa Meio Ambiente, Rodovia SP-340, Km 127,5, Tanquinho Velho, Caixa Postal 69, CEP 13820-000 Jaguariúna, SP, Brasil. E-mail: alfredo.luiz@embrapa.br
\end{abstract}

Resumo - O objetivo deste trabalho foi desenvolver um método para identificação e monitoramento, em tempo quase real, de áreas agrícolas cultivadas com lavouras temporárias de verão, com uso de imagens orbitais Modis, no Estado do Rio Grande do Sul. A metodologia foi denominada detecção de áreas agrícolas em tempo quase real (DATQuaR) e utiliza imagens do sensor Modis referentes aos índices de vegetação (IVs) EVI e NDVI, disponibilizadas em composições de 16 dias. Foram utilizadas quatro métricas para agregar os valores de IVs por pixel, dentro dos períodos bimensais avaliados: média, máximo, mínimo e mediana. Para gerar as imagens (ImDATQuaR), a imagem agregada para o período imediatamente anterior foi subtraída da imagem agregada para o período em monitoramento. Essas imagens foram classificadas por meio de fatiamento e comparadas às classes de referência obtidas pela interpretação visual de pixels aleatorizados em imagens Landsat. Cada ImDATQuaR gerou dois mapas DATQuaR: um com filtragem de moda com janela 3x3 pixels e outro sem filtragem. O melhor mapa DATQuaR é produzido com uso de imagens EVI e filtragem - ao se subtrair a imagem de mínimo valor para o período anterior da imagem de máximo valor para o período monitorado - e atinge concordâncias com a referência superiores a $81 \%$.

Termos para indexação: DATQuaR, mapas de culturas de verão, monitoramento agrícola, sensoriamento remoto.

\section{Near real-time detection of crop fields with Modis images}

\begin{abstract}
The objective of this work was to develop a method to identify and monitor, in near real-time, crop field areas cultivated with temporary summer crops, using Modis orbital images, in the state of Rio Grande do Sul, Brazil. The methodology was called near real-time detection of crop fields (DATQuaR) and uses Modis sensor images of the NDVI and EVI vegetation indices (VIs) from 16-day composites. Four different metrics were used to aggregate the values of VIs per pixel, in the bimonthly periods evaluated: average, maximum, minimum, and median. To generate the images (ImDATQuaR), the aggregated image for the previous period was subtracted from the aggregated image for the monitored period. These images were classified by slicing and compared with the reference classes obtained by the visual interpretation of randomly selected pixels in Landsat images. Each ImDATQuaR image generated two DATQuaR maps: one with a 3x3 pixel window mode filter and another without filtering. The best DATQuaR map is produced using EVI images and filtering - by subtracting the image of minimum value for the previous period from the image of maximum value for the monitored period - and achieves agreement with the reference over $81 \%$.
\end{abstract}

Index terms: DATQuaR, summer crop maps, crop monitoring, remote sensing.

\section{Introdução}

A maioria das metodologias atualmente utilizadas para o monitoramento e a estimativa de área ocupada com culturas agrícolas, a partir de imagens orbitais, gera mapas como produto final (McRoberts, 2011). O monitoramento da localização de culturas agrícolas por imagens de sensoriamento remoto pode ser caracterizado como a produção de cenários das condições de culturas agrícolas visíveis em imagens orbitais, em intervalos regulares e inferiores à duração da safra, para gerar informações com elevado valor (Atzberger, 2013; Kouadio et al., 2014; Sakamoto et al., 2014). 
A geração de informações que ocorrem mais de uma vez durante a safra e a sua disponibilização dentro do período ao qual elas fazem referência pode ser considerada como "em tempo quase real". Uma das características favoráveis do uso de imagens de sensoriamento remoto é a possibilidade de se produzir informações sobre a localização e a área ocupada com as culturas durante a safra (Atzberger, 2013). Porém, a produção de informações desse tipo com o uso de imagens Landsat-likeé uma tarefa de alta complexidade, especialmente em razão da presença recorrente de cobertura de nuvens (Asner, 2001; Sugawara et al., 2008; Eberhardt et al., 2014). Nesse contexto, a opção mais adequada é utilizar produtos com maior resolução temporal, como as imagens do sensor "moderate resolution imaging spectroradiometer" (Modis), que permitem o recobrimento de todo o planeta, a cada 2 dias, entre os paralelos $30^{\circ}$, e, diariamente, entre os paralelos $30^{\circ}$ e os polos (Justice et al., 1998).

Os dados Modis geralmente são utilizados para identificação da resposta espectro-temporal das culturas agrícolas, com base na alta resolução temporal das imagens. Abordagens desse tipo tem ganhado destaque (Bernardes et al., 2011), principalmente quanto ao uso de índices de vegetação (IVs), em períodos específicos ao longo da safra (Rizzi et al., 2009; Gusso et al., 2012; Johann et al., 2012; Risso et al., 2012). No entanto, grande parte das metodologias desenvolvidas gera apenas um mapa para cada safra, geralmente disponível somente após o início da colheita da safra ou mesmo ao final da safra. Eberhardt et al. (2013) adotaram o "crop enhacement index - preview estimate" (CEI-PE) para identificar, de forma precoce, áreas cultivadas com soja no Estado de Mato Grosso. Em 11 safras, os autores conseguiram alcançar exatidão espacial média de: 91,6\%, com 16 dias de antecipação dos mapas; 82,8\%, com 32 dias de antecipação; e 73,9\%, com 48 dias de antecipação, em comparação à metodologia proposta por Rizzi et al. (2009).

O acesso aos mapas de culturas da safra, durante a sua ocorrência, é ferramenta de elevado potencial para os envolvidos na cadeia de produtores agrícolas. Mapas de cultura produzidos durante a safra podem ser utilizados para identificação de possíveis gargalos logísticos, como regiões sob ameaça de problemas sanitários, e de respeito ou não ao vazio sanitário, bem como para estimação do volume de produção a ser escoado, e para identificação do andamento da safra e da localização das lavouras, por exemplo, por instituições governamentais, entre outros possíveis usos.

A geração de mapas de cultura "em tempo quase real", no presente trabalho, refere-se à produção e à disponibilização de mapas em intervalos regulares de 2 meses. As culturas de soja e milho foram consideradas de relevância para o monitoramento durante a safra, já que ocupam a maior parte das áreas agrícolas no Estado do Rio Grande do Sul, com mais de 5,5 milhões de hectares (Companhia Nacional de Abastecimento, 2014), e apresentam ciclos e épocas de desenvolvimento similares, além de importância econômica.

O objetivo deste trabalho foi desenvolver um método para identificação e monitoramento em tempo quase real, de áreas agrícolas cultivadas com lavouras temporárias de verão, com uso de imagens orbitais Modis, no Estado do Rio Grande do Sul.

\section{Material e Métodos}

A região de estudo compreende o Estado do Rio Grande do Sul, situado entre as latitudes 27 e $34^{\circ} 0^{\prime} \mathrm{S}$, e as longitudes 49 e $58^{\circ} 0^{\prime} \mathrm{W}$ (Figura 1). O estado é um importante produtor de soja (Glycine $\max$ L.) e de milho (Zea mays L.), e responde, respectivamente, por 15,6 e 16,3\% da área cultivada no Brasil (Companhia Nacional de Abastecimento, 2014). Boa parte dessas culturas está concentrada na mesorregião Noroeste Rio-grandense, com aproximadamente $58,8 \%$ da área cultivada no RS (Instituto Brasileiro de Geografia e Estatística, 2015). A soja e o milho apresentam épocas de cultivo semelhantes (Cunha et al., 2001; Maluf et al., 2001). No caso da soja, outubro marca o início da semeadura, que se estende até dezembro (Cunha et al., 2001); nessas condições, é possível detectar os culturas de soja, em pleno vigor vegetativo, entre dezembro e março, nas imagens de sensoriamento remoto. Já o início da semeadura do milho ocorre a partir de agosto e pode se estender até janeiro (Maluf et al., 2001), com ciclo médio de 120 dias, o que permite visualizá-lo em imagens de sensoriamento remoto de novembro a abril.

Ao se considerar as similaridades entre as culturas de soja e milho nas imagens de IVs e a predominância dessas duas culturas no Rio Grande do Sul, determinouse que a soja e o milho seriam analisados de modo conjunto, como uma classe única chamada soja + milho. 
A metodologia DATQuaR utiliza imagens processadas MOD13Q1, correspondentes a dois diferentes IVs, disponibilizadas em composições de 16 dias e com resolução espacial de $250 \mathrm{~m}$, elaboradas a partir de imagens diárias adquiridas pelo sensor Modis da plataforma Terra. Os dois IVs utilizados foram o "normalized difference vegetation index" (NDVI) (Rouse et al., 1973) e o "enhanced vegetation index" (EVI) (Huete et al., 1997), disponibilizados pelo programa Earth Observation System (EOS) (Huete et al., 1999).

Em virtude das características dos talhões agrícolas e da dinâmica das culturas, é necessário um conjunto de imagens orbitais livres de nuvens para identificá-los em intervalos regulares. Essas condicionantes tornam a elaboração de mapas de culturas nas condições de um estado como o Rio Grande do Sul um desafio (Rizzi \& Rudorff, 2005; Gusso et al., 2012) e justificam o uso de imagens Modis.
Foram elaborados mapas de culturas para quatro períodos bimensais de monitoramento, para a safra de verão 2013/2014, entre setembro de 2013 e abril de 2014. Portanto, os mapas produzidos identificam os talhões de soja e milho para os períodos de setembro/ outubro de 2013, novembro/dezembro de 2013, janeiro/fevereiro de 2014 e março/abril de 2014. Toda a metodologia DATQuaR foi implementada em nível de script no programa R (R Development Core Team, 2014).

Para a detecção de áreas de culturas de verão, foram utilizados os incrementos de IVs entre os períodos bimensais, tendo-se explorado duas características peculiares das culturas agrícolas de verão: velocidade de acúmulo e sazonalidade de presença de biomassa, como pressuposto em outros trabalhos (Rizzi et al., 2009; Gusso et al., 2012; Johann et al., 2012; Risso et al., 2012; Eberhardt et al., 2013). As composições MOD13Q1 de 16 dias foram agrupadas dentro dos

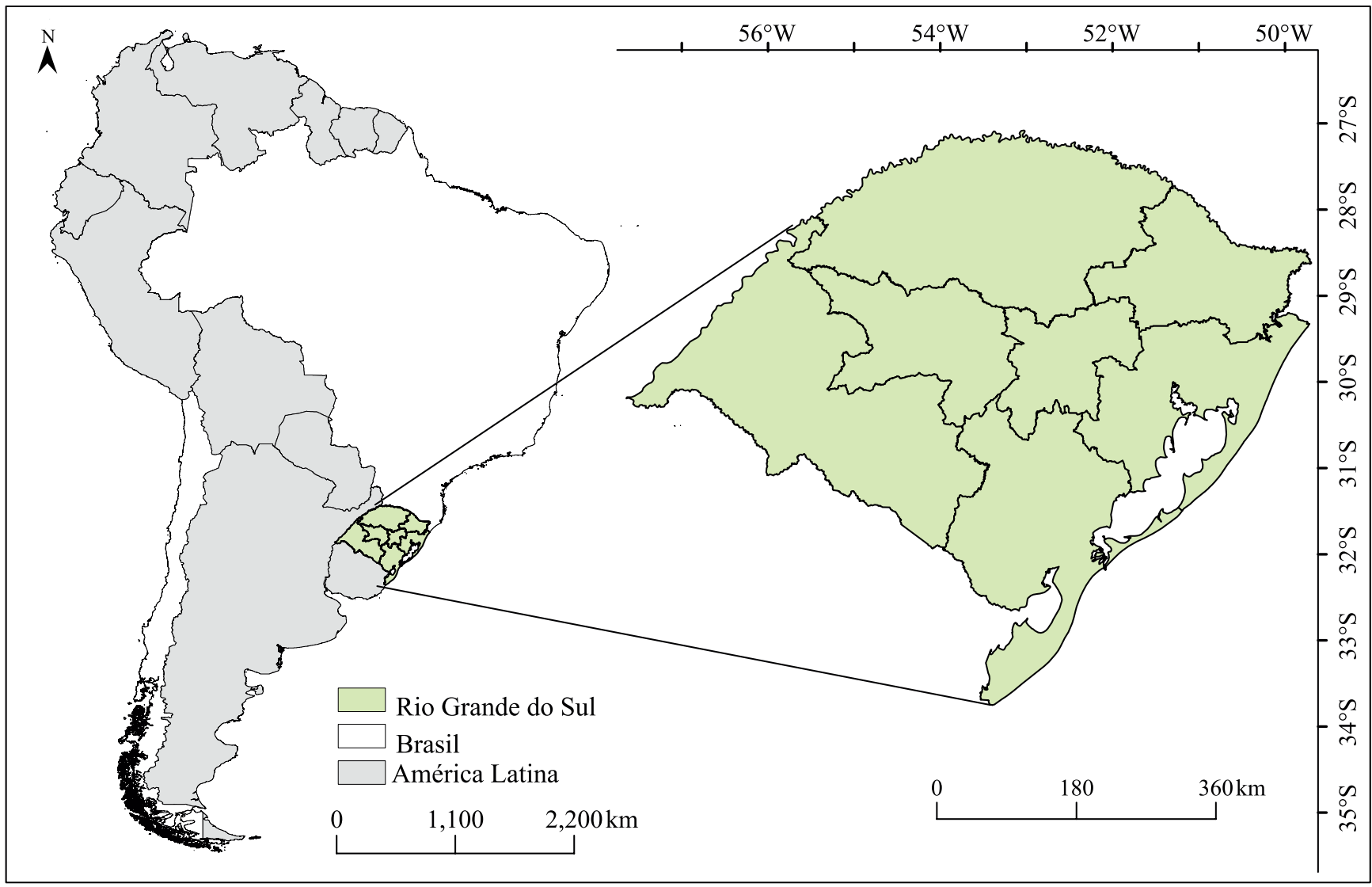

Figura 1. Região de estudo, que compreende o Estado do Rio Grande do Sul (em verde). Subdivisão do estado de acordo com as mesorregiões definidas pelo Instituto Brasileiro de Geografia e Estatística (Instituto Brasileiro de Geografia e Estatística, 1990). 
períodos bimensais, para formação das imagens de cada período. Por exemplo, para formar a imagem agregada do período de janeiro/fevereiro, foram utilizadas as composições MOD13Q1 com data inicial no dia 1 do ano 1 (composição de 1 a 16 de janeiro), assim como as imagens 017 (de 17 de janeiro e 1 de fevereiro) e 033 (de 2 de fevereiro a 18 de fevereiro). No processo de agregação dentro dos períodos bimensais, utilizaram-se as imagens NDVI e EVI separadamente. As imagens agregadas de cada período foram elaboradas por pixel, pela aplicação de quatro diferentes métricas para escolha do valor a ser inserido na nova imagem: mínimo valor de IVs por pixel (MinIVs); máximo valor de IVs por pixel (MaxIVs); média do valor de IVs por pixel (MeanIVs); e mediana do valor de IVs por pixel (MedIVs).

Dessa forma, foram elaboradas duas imagens agregadas - uma de NDVI e outra de EVI - para cada uma das quatro métricas, em cada período bimensal, o que totalizou oito imagens agregadas para cada período: MinEVI e MinNDVI; MaxEVI e MaxNDVI; MeanEVI e MeanNDVI; e MedEVI e MedNDVI.
As imagens agregadas dentro do período bimensal foram utilizadas para a formação da imagem DATQuaR (ImDATQuaR), que representa o incremento de valores de IVs diretamente relacionados aos incrementos de biomassa. As imagens ImDATQuaR foram elaboradas por meio da subtração de duas imagens agregadas por período bimensal. Por exemplo, para o período em monitoramento de janeiro/fevereiro de 2014, a identificação das áreas de soja+milho por imagens Modis foi feita a partir da subtração da imagem agregada de janeiro/fevereiro de 2014 (img1) da imagem agregada de novembro/dezembro de 2013 (img0) (ImDATQuaR = img1 - img0). Os incrementos de IVs entre os dois períodos bimensais representam os incrementos em biomassa detectados nas imagens Modis, no período em monitoramento.

Foram elaboradas 48 diferentes imagens ImDATQuaR para cada período bimensal, a partir das imagens agregadas para cada período, elaboradas com uso de imagens NDVI e EVI, produzidas com as métricas mínimo, máximo, média e mediana (Tabela 1). As diferenças entre as imagens agregadas

Tabela 1. Imagens de índices de vegetação agregadas para cada período. As imagens de detecção de áreas agrícolas em tempo quase real foram formadas a partir da diferença das imagens agregadas em cada período bimensal.

\begin{tabular}{|c|c|c|c|c|c|c|}
\hline \multirow{2}{*}{$\begin{array}{l}\text { Período anterior ao de } \\
\text { monitoramento }^{(1)}\end{array}$} & \multicolumn{6}{|c|}{ Período em monitoramento $^{(2)}$} \\
\hline & MaxEVI & MaxNDVI & MedEVI & MedNDVI & MeanEVI & MeanNDVI \\
\hline \multirow{3}{*}{ MinEVI } & MaxEVI & MaxNDVI & MedEVI & MedNDVI & MeanEVI & MeanNDVI \\
\hline & - & - & - & - & - & - \\
\hline & MinEVI & MinEVI & MinEVI & MinEVI & MinEVI & MinEVI \\
\hline \multirow{3}{*}{ MinNDVI } & MaxEVI & MaxNDVI & MedEVI & MedNDVI & MeanEVI & MeanNDVI \\
\hline & - & - & - & - & - & - \\
\hline & MinNDVI & MinNDVI & MinNDVI & MinNDVI & MinNDVI & MinNDVI \\
\hline \multirow{3}{*}{ MaxEVI } & MaxEVI & MaxNDVI & MedEVI & MedNDVI & MeanEVI & MeanNDVI \\
\hline & - & - & - & - & - & - \\
\hline & MaxEVI & MaxEVI & MaxEVI & MaxEVI & MaxEVI & MaxEVI \\
\hline \multirow{3}{*}{ MaxNDVI } & MaxEVI & MaxNDVI & MedEVI & MedNDVI & MeanEVI & MeanNDVI \\
\hline & - & - & - & - & - & - \\
\hline & MaxNDVI & MaxNDVI & MaxNDVI & MaxNDVI & MaxNDVI & MaxNDVI \\
\hline \multirow{3}{*}{ MedEVI } & MaxEVI & MaxNDVI & MedEVI & MedNDVI & MeanEVI & MeanNDVI \\
\hline & - & - & - & - & - & - \\
\hline & MedEVI & MedEVI & MedEVI & MedEVI & MedEVI & MedEVI \\
\hline \multirow{3}{*}{ MedNDVI } & MaxEVI & MaxNDVI & MedEVI & MedNDVI & MeanEVI & MeanNDVI \\
\hline & - & - & - & - & - & - \\
\hline & MedNDVI & MedNDVI & MedNDVI & MedNDVI & MedNDVI & MedNDVI \\
\hline \multirow{3}{*}{ MeanEVI } & MaxEVI & MaxNDVI & MedEVI & MedNDVI & MeanEVI & MeanNDVI \\
\hline & - & - & - & - & - & - \\
\hline & MeanEVI & MeanEVI & MeanEVI & MeanEVI & MeanEVI & MeanEVI \\
\hline \multirow{3}{*}{ MeanNDVI } & MaxEVI & MaxNDVI & MedEVI & MedNDVI & MeanEVI & MeanNDVI \\
\hline & - & - & - & - & - & - \\
\hline & MeanNDVI & MeanNDVI & MeanNDVI & MeanNDVI & MeanNDVI & MeanNDVI \\
\hline
\end{tabular}

(1)Nas linhas, estão as imagens agregadas para o período anterior ao de monitoramento. ${ }^{(2)}$ Nas colunas, estão as imagens agregadas para o período de monitoramento, para o qual foram gerados os mapas DATQuaR. NDVI, "normalized vegetation index"; EVI, "enhanced vegetation index". 
de EVI e NDVI podem ser testadas, uma vez que, para um mesmo alvo, há tendência de os valores para EVI serem inferiores aos para NDVI (Huete et al., 1999). Assim, para os casos de talhões não cultivados com soja ou milho, há tendência de os valores serem reduzidos ao se subtrair de uma imagem agregada de EVI outra imagem agregada de NDVI. Essa resposta já foi observada por Risso et al. (2012). O contrário também foi relatado para casos nos quais foram subtraídas das imagens agregadas de NDVI uma imagem agregada de EVI, em que a amplitude tende a ser maior do que quando se utiliza somente um dos IVs. Contudo, essa possibilidade é pouco explorada na literatura.

Posteriormente, as imagens ImDATQuaR resultantes de todas as combinações possíveis foram classificadas por meio de fatiamento, tendo-se definido um limiar que produzisse a área identificada como cultura no mapa DATQuaR. Essa área foi a mais próxima possível daquela indicada para cada mesorregião, obtida por meio da aleatorização de um conjunto de 5.000 pixels Landsat de imagens adquiridas pelo sensor "operational land imager" (OLI), para cada um dos quatro períodos bimensais de monitoramento. Os pixels foram obtidos por amostragem aleatória seguindo os critérios definidos por Luiz et al. (2012) e foram classificados por interpretação visual, tendo-se utilizado a composição colorida RGB-564 de imagens adquiridas pelo sensor Landsat/OLI. Assim, foram gerados quatro conjuntos de 5.000 pixels aleatorizados: um para cada período bimensal de estimativa.

Para cada um dos conjuntos de 5.000 pixels aleatorizados, foram considerados os pixels classificados como soja, milho e outros; os pixels classificados como nuvem foram excluídos. As classes soja e milho foram reagrupadas em uma única classe ( $s o j a+m i l h o$ ), e a classe outros foi mantida com o mesmo rótulo, como referência para análise dos mapas DATQuaR.

Os conjuntos de pixels amostrados e classificados por interpretação visual serviram de referência para todo o método DATQuaR, desde a calibração (valores de área ocupada com soja+milho por período, em cada mesorregião) até a avaliação da concordância global (comparação entre os pixels aleatorizados e os mapas DATQuaR). A metodologia utilizada para a classificação visual dos pixels aleatorizados foi avaliada por meio de uma campanha de campo realizada entre 9 e 15 de fevereiro de 2014. A partir da campanha de campo, foi definida uma exatidão de classificação dos pixels aleatorizados da ordem de $95,74 \%$.

Para cada uma das 48 imagens ImDATQuaR para cada período, foram gerados dois mapas DATQuaR: um que, após o fatiamento, foi submetido à filtragem de moda com janela $3 \times 3$ pixels; e outro gerado apenas com o fatiamento, sem a aplicação do processo de filtragem. Ambos os mapas DATQuaR, gerados com e sem filtragem, produzidos a partir da mesma imagem ImDATQuaR, apresentaram valores de área identificada como soja + milho similares entre si (diferenças inferiores a $\pm 0,5 \%$ ). Cabe ressaltar que a aplicação de filtragem de moda tende a retirar do mapa final pixels classificados erroneamente, seja por imperfeições de origem radiométrica na formação das imagens Modis ou por inserção no processo de elaboração das composições MOD13Q1, em razão de problemas de seleção de pixel com ruído (Rizzi et al., 2009); também tende a minimizar a classificação de pixels isolados, ao se considerar a tendência de as áreas agrícolas serem cultivadas em agrupamentos.

$\mathrm{O}$ processo de classificação foi implementado em uma rotina interativa na linguagem do programa $R$, que reclassificou cada imagem ImDATQuaR, com aumento gradual dos valores de limiar até que a área identificada no mapa DATQuaR apresentasse diferença relativa entre $\pm 0,5$ e $0 \%$, em comparação à obtida por meio de amostragem em imagens Landsat. $\mathrm{O}$ intervalo $\pm 0,5 \%$ foi adotado, ao se considerar que, em algumas situações, ao se aplicar uma variação de unidade do limiar de classificação, a área identificada no mapa DATQuaR será maior que o valor de área almejado.

Assim, nos casos em que o processo de filtragem não foi aplicado para geração dos mapas DATQuaR, após a classificação por fatiamento, o algoritmo de classificação encerra a busca pelo melhor limiar quando o mapa gerado apresenta-se dentro deste limite de diferença de área de culturas $( \pm 0,5 \%)$; o mapa produzido é, então, salvo. Para os mapas DATQuaR que passaram pelo processo de filtragem, o mesmo procedimento foi realizado após o fatiamento, com cálculo de área após a filtragem e algoritmo encerrado a partir da mesma regra de diferença máxima de $\pm 0,5 \%$.

Para a avaliação dos mapas DATQuaR a partir da referência para cada período, foram comparadas as classes dos pixels aleatorizados na referência e dos pixels coincidentes geograficamente nos mapas DATQuaR de seus respectivos períodos. De acordo 
com Congalton \& Green (1999), na matriz de confusão para cada mapa DATQuaR, a diagonal representa o número de observações coincidentes para as classes e as extremidades, que referem-se às exatidões de produtor e consumidor. Os valores de concordância global por mesorregião do RS foram obtidos a partir da divisão da soma do número de observações coincidentes (diagonal da matriz de confusão) pelo número total de observações contabilizadas (determinado pelo número de pixels Landsat/OLI aleatorizados).

Os valores de concordância global computados em nível de mesorregião foram posteriormente agregados em nível estadual, por meio da elaboração da média ponderada entre a concordância global de cada mesorregião e a proporção de ocorrência de área ocupada com soja+milho em cada mesorregião, na área total ocupada no RS, determinada pelo processo de amostragem de pixels Landsat/OLI. Em seguida, os valores de concordância global em nível estadual foram ranqueados para cada período, para as diferentes formas de geração dos mapas DATQuaR. A partir desse ranqueamento, foi identificada a moda para o posicionamento em termos de concordância global de cada forma de geração de mapas DATQuaR, o que possibilita definir qual o melhor formato de geração de mapas DATQuaR para identificação de soja+milho no Estado do RS, na safra 2013/2014.

\section{Resultados e Discussão}

Os mapas DATQuaR mostram as diferenças observadas para um mesmo período de janeiro e fevereiro de 2014, com diferentes ImDATQuaR (Figura 2). Os vetores representam os mapas DATQuaR sobrepostos à imagem Landsat 8/OLI, com composição RGB-564, para facilitar a visualização das áreas de soja e milho.

Esses recortes mostram os mapas obtidos pelas diferentes métricas, utilizados para gerar imagens agregadas de IVs, para elaboração das imagens ImDATQuaR. Apesar de os mapas DATQuaR utilizarem o mesmo índice de vegetação (EVI) e a mesma diferença simples entre as imagens dos períodos para geração da ImDATQuaR, há variações visíveis entre eles.

Nos casos dos recortes $\mathrm{C}$ e D (Figura 2), há diferenças entre o mapeamento dos talhões, especialmente no caso dos talhões pertencentes à classe outros. Vale ressaltar que essas diferenças são decorrentes da métrica utilizada para agregar as imagens dentro do período bimensal; portanto, fica evidente a importância de se definir qual métrica pode produzir mapas DATQuaR de melhor qualidade, pela melhor delimitação dos talhões.

$\mathrm{O}$ recorte $\mathrm{E}$ indica que os talhões de arroz (polígonos em marrom) da região Sul do RS não foram erroneamente mapeados como soja+milho (Figura 2). Em parte, era esperado que uma porção das áreas de cultivo de arroz irrigado (Oryza sativa L.) fosse inserida erroneamente nos mapas DATQuaR, dado que o cultivo do arroz também apresenta incrementos de IVs elevados (Klering et al., 2011; Klering et al., 2013). Porém, na formatação da metodologia DATQuaR, para calibração do fatiamento, são utilizados valores de área ocupada obtidos com base nas estimativas por amostragem probabilística em imagens Landsat. Esse formato favorece a redução de possíveis erros de inclusão de áreas de arroz e de exclusão de áreas de soja + milho, já que, nessas condições, o algoritmo de classificação buscará selecionar os talhões de maior incremento de IVs e que totalizem uma área ocupada de soja + milho equivalente à observada na referência (Rizzi et al., 2009; Risso et al., 2012).

Os valores de concordância global foram computados a partir da aplicação da metodologia DATQuaR nas diferentes formas de geração das imagens ImDATQuaR. Esses valores indicam que a configuração dos melhores resultados foi a que utilizou o MinIVs para o período anterior ao em monitoramento. Essa resposta pode ser explicada pelas maiores diferenças relativas entre as classes soja + milho e outros (intervalo maior da primeira) com a utilização da imagem de mínimo valor para o período anterior ao em monitoramento.

Como a metodologia implementada se baseia na identificação do incremento dos valores de IVs entre dois períodos diferentes, o formato que utiliza MinIVs no período anterior ao em monitoramento difere dos adotados em trabalhos que buscavam identificar talhões agrícolas a partir dos incrementos de IVs (Gusso et al., 2012; Risso et al., 2012). Assim, ao se considerar os resultados anteriormente apresentados, serão discutidos somente àqueles obtidos com a utilização de MinIVs na formatação da metodologia DATQuaR.

Os recortes mostram as diferenças na identificação dos talhões de cultura de soja + milho, em função de três das métricas utilizadas (máximo, média e mediana) para geração das imagens ImDATQuaR, para o período em 
monitoramento (Figura 2). Os três vetores apresentam convergência entre si, com partes coincidentes tanto nos talhões maiores quanto nos menores. No entanto, em muitos casos, as métricas de agregação MedEVI e MeanEVI apresentaram os menores talhões mapeados erroneamente. Sob a ótica da identificação dos talhões de soja+milho, estes foram definidos de modo mais preciso quando utilizou-se a métrica MaxEVI para o período de estimativa (vetor em preto). Isso pode ser constatado ao se visualizar as áreas em alaranjado, de talhões de soja em imagens Landsat 8/OLI, que foram corretamente delimitadas com esse formato (Figura 2). Resultados semelhantes foram obtidos por Risso et al. (2012) e Rizzi et al. (2009), os quais verificaram que a utilização do MaxEVI foi capaz de identificar os talhões agrícolas com maior exatidão.
A análise para determinação da concordância global entre os mapas DATQuaR sem utilização de filtragem de moda mostrou que estes não diferiram entre si para cada período, quando comparados os diferentes métodos de formação das imagens ImDATQuaR em um mesmo período (Tabela 2). Os valores de concordância global evidenciaram que, nos mapas DATQuaR sem uso de filtragem de moda, o formato que produziu os melhores valores de concordância global nos três períodos, com maior presença de culturas agrícolas de novembro/ dezembro de 2013 a março/abril de 2014, foi aquele que utilizou a diferença simples entre a imagem de máximos valores de EVI, para o período monitorado, e a imagem de mínimos valores de NDVI para o período prévio (Tabela 2).

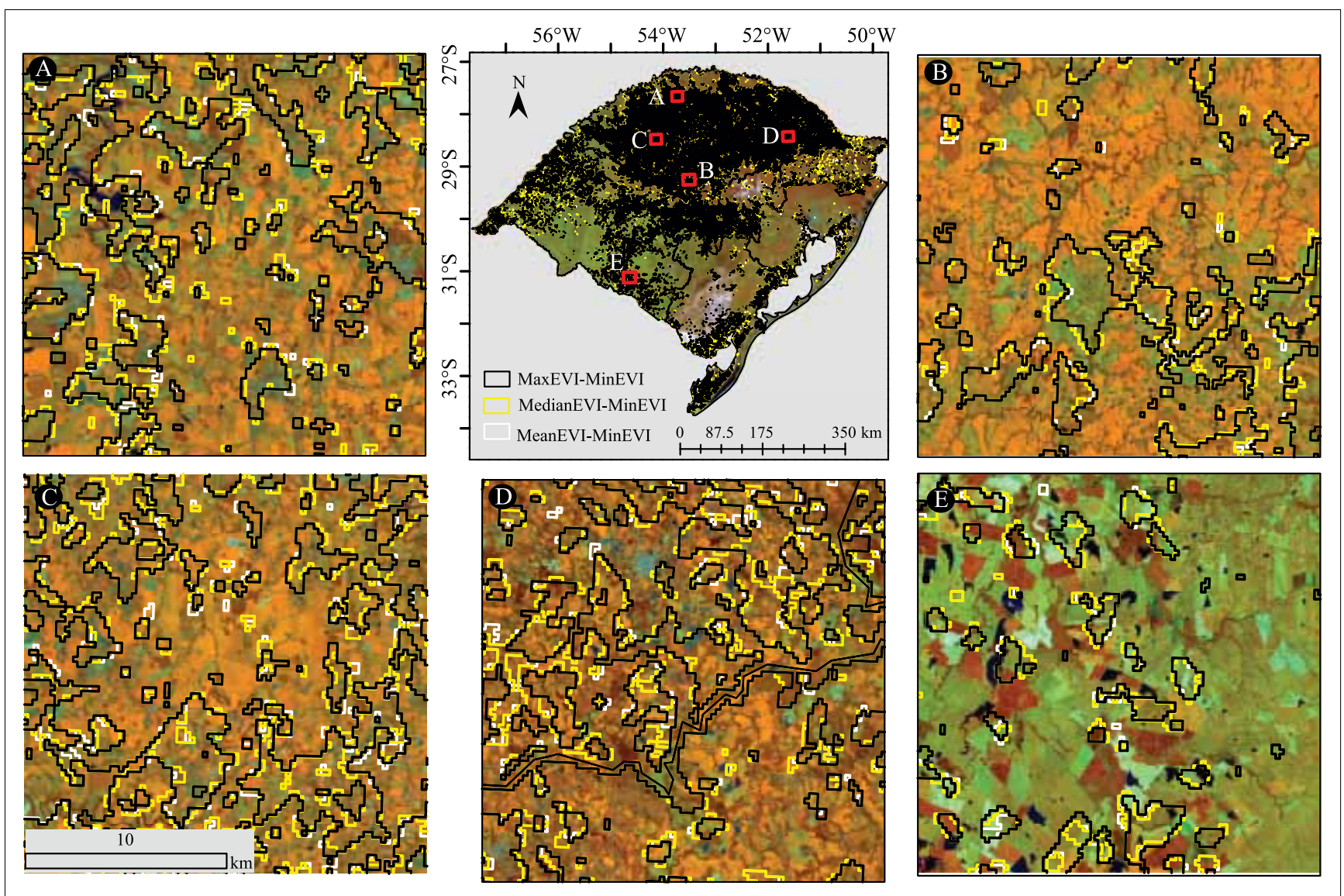

Figura 2. Mapas de detecção de áreas agrícolas em tempo quase real (DATQuaR) (vetores), no período de janeiro/fevereiro de 2014, sobrepostos a imagens Landsat 8/OLI, para visualização dos talhões mapeados. O mapeamento difere de acordo com as formas de agregar as imagens ImDATQuaR. Todos os mapas contidos nessa Figura passaram pelo processo de filtragem de moda com janela 3x3 pixels. MaxEVI-MinEVI, vetor em preto; MedEVI-MinEVI, vetor em amarelo; e MeanEVI-MinEVI, vetor em branco. NDVI, "normalized vegetation index"; EVI, "enhanced vegetation index". 
Esse resultado difere do observado por Risso et al. (2012) para o Estado do Mato Grosso, onde foi possível, por meio do uso de uma imagem agregada de NDVI para o período anterior ao de monitoramento, produzir melhores resultados de acerto de mapeamento e de discriminação dos alvos agrícolas em relação aos demais. Contudo, em vez da metodologia DATQuaR, Risso et al. (2012) utilizaram a filtragem de moda.

No entanto, quando adotou-se a filtragem de moda nos mapas DATQuaR, o método de formação das imagens ImDATQuaR de maior concordância global foi aquele que utilizou apenas imagens EVI na configuração de seleção do máximo valor observado por pixel, para o período em monitoramento, e do mínimo valor para o período prévio (Tabela 3). Os valores de concordância nos períodos bimensais de menor acerto, de janeiro/ fevereiro a março/abril de 2014 (Tabelas 2 e 3), foram superiores aos observados sem aplicação do processo de filtragem.

Quanto aos resultados de concordância global obtidos entre os mapas DATQuaR e os pixels aleatorizados sobre imagens Landsat 8, cabe reforçar que, para o período de setembro/outubro de 2013, apenas três mesorregiões apresentaram soja+milho cultivados: Noroeste Rio-grandense, Centro Ocidental Rio-grandense e Sudeste Rio-grandense. Os valores de concordância (Tabelas 2 e 3) para esse período foram

Tabela 2. Valores de concordância global, em nível estadual, dos mapas de detecção de áreas agrícolas em tempo quase real (DATQuaR), sem filtragem e com pixels Landsat 8 classificados por interpretação visual.

\begin{tabular}{|c|c|c|c|c|}
\hline Mapas DATQuaR $^{(1)}$ & $\begin{array}{c}\text { Set./out. } \\
2013\end{array}$ & $\begin{array}{c}\text { Nov./dez. } \\
2013 \\
\end{array}$ & $\begin{array}{c}\text { Jan./fev. } \\
2014\end{array}$ & $\begin{array}{c}\text { Mar./abr. } \\
2014\end{array}$ \\
\hline MaxEVI - MinEVI & 99,43 & 87,10 & 81,02 & 80,46 \\
\hline MeanEVI - MinEVI & 99,35 & 87,20 & 80,73 & 78,79 \\
\hline MedEVI - MinEVI & 99,35 & 87,11 & 80,78 & 79,15 \\
\hline MaxNDVI - MinEVI & 99,46 & 86,69 & 80,36 & 79,61 \\
\hline MeanNDVI - MinEVI & 99,42 & 85,92 & 78,77 & 72,81 \\
\hline MedNDVI - MinEVI & 99,42 & 86,45 & 79,52 & 75,51 \\
\hline MaxEVI - MinNDVI & 99,39 & 90,99 & 81,63 & 80,93 \\
\hline MeanEVI - MinNDVI & 99,42 & 91,59 & 80,94 & 78,72 \\
\hline MedEVI - MinNDVI & 99,39 & 91,60 & 80,88 & 79,14 \\
\hline MaxNDVI - MinNDVI & 99,46 & 87,99 & 80,61 & 80,08 \\
\hline MeanNDVI - MinNDVI & 99,38 & 87,78 & 80,00 & 77,10 \\
\hline MedNDVI - MinNDVI & 99,46 & 87,96 & 80,07 & 78,37 \\
\hline
\end{tabular}

${ }^{(1)} \mathrm{O}$ sinal entre as imagens para cada período identifica a diferença simples entre elas. NDVI, "normalized vegetation index"; EVI, "enhanced vegetation index". calculados somente com as ocorrências dessas três mesorregiões. É importante destacar que o cálculo da concordância global em nível estadual elaborado - ao se ponderar o acerto do percentual da área ocupada com soja + milho que cada uma das mesorregiões representa no total do Estado, em função da área ocupada identificada por amostragem de pixel Landsat 8 - tende a reduzir os impactos do acerto de mesorregiões menos importantes em termos de área ocupada. Isso reforça a representatividade da concordância global de acordo com as mesorregiões mais representativas em termos de área ocupada.

Os valores de concordância global foram menores à medida que os valores de área, identificados para cada período de monitoramento por amostragem de pixels Landsat 8, mostraram maiores áreas ocupadas. A sequência de área ocupada com soja + milho para os períodos bimensais, em ordem decrescente de valores, foi: janeiro/fevereiro, março/abril, novembro/ dezembro e setembro/outubro.

As diferenças entre os valores de concordância global obtidos para os mapas DATQuaR produzidos com o processo de filtragem e para aqueles que não passaram por filtragem foram de, no máximo, 3\% (Tabela 4). Nessa comparação, os valores de concordância global para os mapas produzidos foram superiores com o processo de filtragem. Essa resposta apenas não foi

Tabela 3. Valores de concordância global, em nível estadual, dos mapas de detecção de áreas agrícolas em tempo quase real (DATQuaR), com filtragem de moda com janela 3x3 e pixels Landsat 8 classificados por interpretação visual.

\begin{tabular}{lcccc}
\hline Mapas DATQuaR $^{(1)}$ & $\begin{array}{c}\text { Set./out. } \\
2013\end{array}$ & $\begin{array}{c}\text { Nov./dez. } \\
2013\end{array}$ & $\begin{array}{c}\text { Jan./fev. } \\
2014\end{array}$ & $\begin{array}{c}\text { Mar./abr. } \\
2014\end{array}$ \\
\hline MaxEVI - MinEVI & 99,53 & 89,22 & 81,24 & 81,38 \\
MeanEVI - MinEVI & 99,46 & 88,81 & 80,92 & 80,13 \\
MedEVI - MinEVI & 99,42 & 88,94 & 80,77 & 80,54 \\
MaxNDVI - MinEVI & 99,63 & 88,78 & 80,12 & 81,37 \\
MeanNDVI - MinEVI & 99,53 & 87,66 & 79,45 & 75,20 \\
MedNDVI - MinEVI & 99,63 & 87,79 & 79,69 & 77,71 \\
MaxEVI - MinNDVI & 99,60 & 91,39 & 80,88 & 80,89 \\
MeanEVI - MinNDVI & 99,63 & 91,26 & 77,58 & 77,83 \\
MedEVI - MinNDVI & 99,63 & 91,37 & 78,27 & 78,33 \\
MaxNDVI - MinNDVI & 99,59 & 89,25 & 80,27 & 81,23 \\
MeanNDVI - MinNDVI & 99,59 & 90,45 & 80,15 & 80,14 \\
MedNDVI - MinNDVI & 99,56 & 90,50 & 80,64 & 80,86 \\
\hline
\end{tabular}

${ }^{(1)} \mathrm{O}$ sinal entre as imagens para cada período identifica a diferença simples entre elas. NDVI, "normalized vegetation index"; EVI, "enhanced vegetation index". 
Tabela 4. Diferença entre os valores de concordância global, em nível estadual, dos mapas de deteç̧ão de áreas agrícolas em tempo quase real (DATQuaR), com e sem filtragem de moda com janela $3 \times 3^{(1)}$.

\begin{tabular}{lcccc}
\hline Mapas DATQuaR $^{(2)}$ & $\begin{array}{c}\text { Set./out. } \\
2013\end{array}$ & $\begin{array}{c}\text { Nov./dez. } \\
2013\end{array}$ & $\begin{array}{c}\text { Jan./fev. } \\
2014\end{array}$ & $\begin{array}{c}\text { Mar./abr. } \\
2014\end{array}$ \\
\hline MaxEVI - MinEVI & 0,10 & 2,12 & 0,22 & 0,92 \\
MeanEVI - MinEVI & 0,11 & 1,61 & 0,19 & 1,35 \\
MedEVI - MinEVI & 0,07 & 1,83 & $-0,01$ & 1,39 \\
MaxNDVI - MinEVI & 0,17 & 2,10 & $-0,24$ & 1,76 \\
MeanNDVI - MinEVI & 0,11 & 1,73 & 0,68 & 2,39 \\
MedNDVI - MinEVI & 0,21 & 1,33 & 0,16 & 2,20 \\
MaxEVI - MinNDVI & 0,21 & 0,40 & $-0,75$ & $-0,04$ \\
MeanEVI - MinNDVI & 0,21 & $-0,33$ & $-3,37$ & $-0,88$ \\
MedEVI - MinNDVI & 0,24 & $-0,23$ & $-2,61$ & $-0,81$ \\
MaxNDVI - MinNDVI & 0,14 & 1,27 & $-0,34$ & 1,15 \\
MeanNDVI - MinNDVI & 0,21 & 2,67 & 0,16 & 3,04 \\
MedNDVI - MinNDVI & 0,10 & 2,53 & 0,58 & 2,49 \\
\hline
\end{tabular}

${ }^{(1)}$ Os valores positivos representam os casos em que os mapas DATQuaR que passaram por processo de filtragem mostraram valores de concordância global superior aos daqueles que não passaram por filtragem; e os valores negativos representam os casos em que os mapas DATQuaR que não passaram pelo processo de filtragem apresentaram valores de concordância maiores do que aqueles sob filtragem. ${ }^{(2)} \mathrm{O}$ sinal entre as imagens para cada período identifica a diferença simples entre elas. NDVI, "normalized vegetation index"; EVI, "enhanced vegetation index".

observada no período em monitoramento de janeiro/ fevereiro de 2014.

Por fim, ao se analisar os valores de concordância entre os mapas DATQuaR, constatou-se que os mapas que passaram pelo processo de filtragem de moda apresentaram valores de concordância mais elevados, quando comparados aos não filtrados (Tabelas 2 e 3 ). Os valores de concordância global para os mapas que passaram pelo processo de filtragem de moda com janela $3 \times 3$ oscilaram entre 75,20 e $99,63 \%$, e para os mapas elaborados sem filtragem, entre 72,81 e $99,46 \%$.

\section{Conclusões}

1. O método detecção de áreas agrícolas em tempo quase real (DATQuaR) possibilita gerar mapas de localização das áreas cultivadas com soja+milho durante a safra de verão, em intervalos regulares de dois meses, o que torna viável o monitoramento da localização das áreas cultivadas com milho (Zea mays) e soja (Glycine max) no Estado do Rio Grande do Sul, durante a safra.

2. O melhor mapa DATQuaR é produzido com uso de imagens EVI e filtragem, ao se subtrair a imagem de mínimo valor para o período anterior da imagem de máximo valor para o período monitorado, e atinge concordâncias com a referência superiores a $81 \%$.

\section{Agradecimentos}

À Coordenação de Aperfeiçoamento de Pessoal de Nível Superior (Capes), pela concessão de bolsa; ao Programa Ciência sem Fronteiras (Projeto 402.597/2012-5) da Capes e do Conselho Nacional de Desenvolvimento Científico e Tecnológico (CNPq), pelo apoio financeiro.

\section{Referências}

ASNER, G.P. Cloud cover in Landsat observations of the Brazilian Amazon. International Journal of Remote Sensing, v.22, p.3855-3862, 2001. DOI: 10.1080/01431160010006926.

ATZBERGER, C. Advances in remote sensing of agriculture: context description, existing operational monitoring systems and major information needs. Remote Sensing, v.5, p.949-981, 2013. DOI: $10.3390 /$ rs5020949.

BERNARDES, T.; ADAMI, M.; FORMAGGIO, A.R.; MOREIRA, M.A.; FRANÇA, D. de A.; NOVAES, M.R. de. Imagens mono e multitemporais Modis para estimativa da área com soja no estado de Mato Grosso. Pesquisa Agropecuária Brasileira, v.46, p.1530-1537, 2011. DOI: 10.1590/S0100-204X2011001100015.

COMPANHIA NACIONAL DE ABASTECIMENTO. Acompanhamento da safra brasileira [de] grãos - décimo segundo levantamento - safra 2013/2014. Brasília: Conab, 2014. v.1, n.12, 126p.

CONGALTON, R.G.; GREEN, K. Assessing the accuracy of remotely sensed data: principles and practices. Boca Raton: CRC Press, 1999.

CUNHA, G.R. da; BARNI, N.A.; HAAS, J.C.; MALUF, J.R.T.; MATZENAUER, R.; PASINATO, A.; PIMENTEL, M.B.M.; PIRES, J.L.F. Zoneamento agrícola e época de semeadura para soja no Rio Grande do Sul. Revista Brasileira de Agrometeorologia, v.9, p.446-459, 2001.

EBERHARDT, I.D.R.; MELLO, M.P.; RIZZI, R.; FORMAGGIO, A.R.; ATZBERGER, C.; LUIZ, A.J.B.; FOSCHIERA, W.; SCHULTZ, B.; TRABAQUINI, K.; GOLTZ, E. Assessment of suitable observation conditions for a monthly operational remote sensing based crop monitoring system. In: GEOSCIENCE AND REMOTE SENSING SYMPOSIUM, 2014, Quebec. Proceedings. New York: IEEE, 2014. p.2126-2129. DOI: 10.1109/ IGARSS.2014.6946886.

EBERHARDT, I.D.R.; RIZZI, R.; RISSO, J.; SANTOS, F.J. dos; FERNANDES, S.L. Mapeamento e estimativa prévia das áreas de soja no Mato Grosso a partir de imagens EVI. In: SIMPÓSIO BRASILEIRO DE SENSORIAMENTO REMOTO, 16., 2013, Foz do Iguaçu. Anais. São José dos Campos: Instituto Nacional de Pesquisas Espaciais, 2013. p.356-366. 
GUSSO, A.; FORMAGGIO, A.R.; RIZZI, R.; ADAMI, M.; RUDORFF, B.F.T. Soybean crop area estimation by Modis/Evi data. Pesquisa Agropecuária Brasileira, v.47, p.425-435, 2012. DOI: 10.1590/S0100-204X2012000300015.

HUETE, A.; JUSTICE, C.; VAN LEEUWEN, W. Modis vegetation index (MOD 13): algorithm theoretical basis document. Version 3. Washington: National Aeronautics and Space Administration, 1999. 129p.

HUETE, A.R.; LIU, H.Q.; BATCHILY, K.; VAN LEEUWEN, W. A comparison of vegetation indices over a global set of TM images for EOS-MODIS. Remote Sensing of Environment, v.59, p.440-451, 1997. DOI: 10.1016/S0034-4257(96)00112-5.

INSTITUTO BRASILEIRO DE GEOGRAFIA E ESTATÍSTICA. Divisão do Brasil em mesorregiões e microrregiões geográficas. Rio de Janeiro, 1990. Disponível em: <http://biblioteca.ibge.gov.br/ visualizacao/monografias/GEBIS\%20\%20RJ/DRB/Divisao $\% 20$ regional_v01.pdf>. Acesso em: 1 nov. 2014.

INSTITUTO BRASILEIRO DE GEOGRAFIA E ESTATÍSTICA. Sistema IBGE de recuperação automática. Banco de dados agregados. Disponível em: <http://www.sidra.ibge.gov.br/bda/>. Acesso em: 8 maio 2015.

JOHANN, J.A.; ROCHA, J.V.; DUFT, D.G.; LAMPARELLI, R.A.C. Estimativa de áreas com culturas de verão no Paraná, por meio de imagens multitemporais EVI/Modis. Pesquisa Agropecuária Brasileira, v.47, p.1295-1306, 2012. DOI: 10.1590/ S0100-204X2012000900015.

JUSTICE, C.O.; VERMOTE, E.; TOWNSHEND, J.R.G.; DEFRIES, R.; ROY, D.P.; HALL, D.K.; SALOMONSON, V.V.; PRIVETTE, J.L.; RIGGS, G.; STRAHLER, A.; LUCHT, W.; MYNENI, R.B.; KNYAZIKHIN, Y.; RUNNING, S.W.; NEMANI, R.R.; ZHENGMING, W.; HUETE, A.R.; VAN LEEUWEN, W.; WOLFE, R.E.; GIGLIO, L.; MULLER, J.-P.; LEWIS, P.; BARNSLEY, M.J. The Moderate Resolution Imaging Spectroradiometer (MODIS): land remote sensing for global change research. IEEE Transactions on Geoscience and Remote Sensing, v.36, p.1228-1249, 1998. DOI: 10.1109/36.701075.

KLERING, E.V.; FONTANA, D.C.; ALVES, R.; ROCHA, J.; BERLATO, M.A. Estimativa de área cultivada com arroz irrigado para o Estado do Rio Grande do Sul a partir de imagens Modis. Ciência e Natura, v.35, p.126-135, 2013.

KLERING, E.V.; WAGNER, A.P.; FONTANA, D.C.; BERLATO, M.A. Comparação entre dois métodos de estimativa de área cultivada com arroz irrigado no Rio Grande do Sul. In: SIMPÓSIO BRASILEIRO DE SENSORIAMENTO REMOTO, 15., 2011, Curitiba. Anais. São José dos Campos: Instituto Nacional de Pesquisas Espaciais, 2011. p.191-198.
KOUADIO, L.; NEWLANDS, N.K.; DAVIDSON, A.; ZHANG, Y.; CHIPANSHI, A. Assessing the performance of MODIS NDVI and EVI for seasonal crop yield forecasting at the ecodistrict scale. Remote Sensing, v.6, p.10193-10214, 2014. DOI: 10.3390/ rs61010193.

LUIZ, A.J.B.; FORMAGGIO, A.R.; EPIPHANIO, J.C.N.; ARENAS-TOLEDO, J.M.; GOLTZ, E.; BRANDÃO, D. Estimativa amostral objetiva de área plantada regional, apoiada em imagens de sensoriamento remoto. Pesquisa Agropecuária Brasileira, v.47, p.1279-1287, 2012. DOI: 10.1590/s0100-204x2012000900013.

MCROBERTS, R.E. Satellite image-based maps: scientific inference or pretty pictures? Remote Sensing of Environment, v.115, p.715-724, 2011. DOI: 10.1016/j.rse.2010.10.013.

MALUF, J.R.T.; CUNHA, G.R. da; MATZENAUER, R.; PASINATO, A.; PIMENTEL, M.B.M.; CAIAFFO, M.R.; PIRES, J.L.F. Zoneamento de riscos climáticos para a cultura de milho no Rio Grande do Sul. Revista Brasileira de Agrometeorologia, v.9, p.460-467, 2001.

R DEVELOPMENT CORE TEAM. R: a language and environment for statistical computing. Vienna: R Foundation for Statistical Computing, 2014.

RISSO, J.; RIZZI, R.; RUDORFF, B.F.T.; ADAMI, M.; SHIMABUKURO, Y.E.; FORMAGGIO, A.R.; EPIPHANIO, R.D.V. Índices de vegetação Modis aplicados na discriminação de áreas de soja. Pesquisa Agropecuária Brasileira, v.47, p.1317-1326, 2012. DOI: 10.1590/S0100-204X2012000900017.

RIZZI, R.; RISSO, J.; EPIPHANIO, R.D.V.; RUDORFF, B.F.T.; FORMAGGIO, A.R.; SHIMABUKURO, Y.E.; FERNANDES, S.L. Estimativa da área de soja no Mato Grosso por meio de imagens MODIS. In: SIMPÓSIO BRASILEIRO DE SENSORIAMENTO REMOTO, 14., 2009, Natal. Anais. São José dos Campos: Instituto Nacional de Pesquisas Espaciais, 2009. p.387-394.

RIZZI, R.; RUDORFF, B.F.T. Estimativa da área de soja no Rio Grande do Sul por meio de imagens Landsat. Revista Brasileira de Cartografia, v.57, p.226-234, 2005.

ROUSE JR, J.W.; HASS, R.H.; DEERING, D.W.; SCHELL, J.A. Monitoring the vernal advancement and retrogradation (green wave effect) of natural vegetation. College Station: Texas A\&M University, 1973.

SAKAMOTO, T.; GITELSON, A.A.; ARKEBAUER, T.J. Near real-time prediction of U.S. corn yields based on time-series MODIS data. Remote Sensing of Environment, v.147, p.219-231, 2014. DOI: 10.1016/j.rse.2014.03.008.

SUGAWARA, L.M.; RUDORFF, B.F.T.; ADAMI, M. Viabilidade de uso de imagens do Landsat em mapeamento de área cultivada com soja no Estado do Paraná. Pesquisa Agropecuária Brasileira, v.43, p.1777-1783, 2008. DOI: 10.1590/S0100-204X2008001200019.

Recebido em 29 de janeiro de 2015 e aprovado em 25 de maio de 2015 